\title{
Incapacidade funcional em mulheres idosas de baixa renda
}

\author{
Functional incapacity among low-income elderly women
}

Jair Sindra Virtuoso Júnior ${ }^{1}$

Ricardo Oliveira Guerra ${ }^{2}$

Instituto de Ciências da Saúde, Universidade Federal do Triângulo Mineiro. Av. Getúlio Guaritá 159, CEA 3 andar, sala 301. 38025-440. Uberaba MG.

jair@ef.uftm.edu.br

${ }^{2}$ Departamento de

Fisioterapia, Universidade

Federal do Rio Grande do

Norte.
Abstract The scope of this study was to analyze the relationship between socio-demographic and health-related aspects and functional incapacity among low-income elderly women. This crosssectional study involved a representative sample of 222 women with mean age of 70 years $( \pm 7.27)$, in community groups in the city of Jequié, in the State of Bahia, Brazil. Anthropometric measurements were taken and interviews staged to examine the socio-demographic, physical and behavioral health variables. The statistical analysis was accomplished with a level of significance of $p<0.05$, with calculation of the respective odds ratio in the binary logistic regression, for analysis of hierarchically-grouped factors. The prevalence of functional incapacity was $46.8 \%$ and, by using hierarchical multivariate analysis, a significant association was detected with increased age, insufficient schooling, hospitalization, lack of physical exercise throughout life and alterations in the cognitive function. The characteristics identified that associated limitations in instrumental activities in daily life suggest a complex causal network in the determination of functional incapacity in low-income elderly women.

Key words Health of the elderly, Socioeconomic factors, Incapacity
Resumo O propósito deste estudo foi o de analisar a relação dos aspectos sociodemográficos e referentes às condições de saúde com a incapacidade funcional em mulheres idosas de baixa renda. O estudo teve delineamento transversal, com uma amostra representativa de 222 mulheres, com média de 70 anos $( \pm 7,27)$, pertencentes a grupos de convivência no município de Jequié, Bahia. Foram realizadas medidas antropométricas e a aplicação de uma entrevista contemplando as variáveis: sociodemográficas, saúde física e comportamental. A análise estatística foi realizada com nível de significância $p<0,05$, com cálculo da respectiva razão de chances na regressão logística binária, para análise de fatores hierarquicamente agrupados. A prevalência de incapacidade funcional foi de 46,8\%, nos quais a partir de análise multivariada hierarquizada verificaram-se associação significativa com o aumento da idade, baixo nível de educação, hospitalização, ausência de práticas de atividades físicas ao longo da vida e alterações na função cognitiva. As características identificadas que associaram as limitações em atividades instrumentais da vida diária sugerem uma complexa rede causal na determinação da capacidade funcional em mulheres idosas de baixa renda.

Palavras-chave Saúde do idoso, Fatores socioeconômicos, Incapacidade 


\section{Introdução}

A capacidade funcional é um determinante da condição de saúde e bem-estar das pessoas. $\mathrm{Na}$ velhice, as funções orgânicas decorrentes do processo de envelhecimento em consequência dos agravos ao longo da vida passam a ser mais vulneráveis ao declínio funcional ${ }^{1}$.

A identificação dos fatores que aceleram o processo natural de perda da capacidade funcional com a idade cronológica é fundamental na adoção de estratégias de saúde mais efetivas, bem como na implementação de políticas públicas que favoreçam a preservação da capacidade funcional das pessoas idosas.

Apesar de haver estudos que sintetizam o processo de perda da capacidade funcional em modelos teóricos ${ }^{2,3}$, há uma necessidade de que tais modelos sejam testados e/ou ajustados às condições de vida das pessoas, em decorrência das particularidades sociais e culturais da região de moradia. Há uma carência de investigações a respeito das condições de vida das populações residentes em regiões mais pobres, principalmente no que diz respeito aos países em desenvolvimento, a exemplo do Brasil, em que as desigualdades sociais são alarmantes.

Assim, o propósito deste estudo foi o de analisar a relação dos aspectos sociodemográficos e referentes às condições de saúde com a condição de dependência na realização das atividades instrumentais da vida diária (AIVDs). Os conhecimentos gerados podem transformar-se em subsídios para a implantação de programas, no planejamento de estratégias de atendimento e intervenção adequados a populações com características similares a este estudo.

\section{Métodos}

Foi realizado um estudo transversal no município de Jequié, localizado a $365 \mathrm{~km}$ de Salvador, capital da Bahia, Região Nordeste do Brasil, no período de outubro a dezembro de 2006. A amostra inicial de 222 mulheres idosas com 60 anos ou mais foi selecionada de forma probabilística e estratificada por grupo de convivência. $\mathrm{O}$ valor da amostra foi proporcional a $42,3 \%$ da população inicial de 528 idosos, que representa o numero total de sujeitos participantes nos 16 grupos de convivência do município de Jequié, estabelecendo-se nível de confiança de $95 \%$ com erro de 5\%.

Para coleta dos dados, foram realizadas medidas de peso e estatura corporal para obtenção do índice de massa corporal (IMC), calculado através do peso corporal $(\mathrm{kg})$ dividido pela estatura (m) ao quadrado. Em seguida aos testes antropométricos, foi aplicada uma entrevista multidimensional no formato face a face. A entrevista utilizada teve sua confiabilidade testada previamente ao estudo, sendo constituída pelos seguintes itens:

- Aspectos sociodemográficos: idade, escolaridade, estado civil, atividade laboral, tamanho da família e nível socioeconômico ${ }^{4}$;

- Aspectos de saúde física: autopercepção de saúde, patologias em conformidade com a Classificação Internacional de Doenças (CID-10) medicamentos em uso contínuo, ocorrências de quedas e número de internamentos nos últimos seis meses;

- Capacidade funcional: atividades instrumentais da vida diária (AIVDs) pelo índice de Lawton $^{6}$, tendo adotado o ponto de corte de 12 pontos para a presença de dependência funcional do tipo moderada a grave;

- Aspectos de saúde mental: na avaliação da função cognitiva, foram utilizados o Short Portable Mental Status Questionare de Pfeifer $\left(\mathrm{SPMQS}^{7}\right.$ e a presença de sintomatologia depressiva medida pela Escala de Depressão Geriátrica GDS-15. Em ambas as escalas, os pontos de corte adotados foram os determinados pela literatura, de acordo com estudos de validação para a população brasileira9,10.

Para confecção do banco de dados, foi utilizado o software Epidata, versão 3.1b, e as análises foram feitas por meio do pacote estatístico SPSS (versão 11). $\mathrm{Na}$ análise descritiva foi feita a distribuição de frequências absolutas, a distribuição percentual, média e desvio padrão (DP). $\mathrm{Na}$ abordagem analítica, foi realizada análise de regressão logística univariada ${ }^{11}$. Para a identificação dos fatores associados com a presença de dependência moderada/grave do idoso, foi realizada análise múltipla de regressão logística ${ }^{11}$, utilizando análise hierarquizada ${ }^{12}$. Mediante a estratégia estabelecida de associações entre as dimensões estudadas (sociodemográfica, saúde física e comportamental), foram elaborados três modelos explicativos de regressão logística binária, introduzindo as variáveis em forma de blocos, permanecendo no modelo subsequente apenas aquelas que tiveram significância estatística $(\mathrm{p}<0,05)$ no modelo anterior.

O critério de saída para todas as variáveis introduzidas em cada modelo foi $\mathrm{p}<0,1$. Ao final, chegou-se a um modelo final de regressão com apenas as variáveis de maior significância 
estatística. O método adotado para introdução das variáveis nos modelos foi o backward stepwi$s e^{12}$. Considerou-se um nível de significância p $<0,05$ e intervalo de confiança (IC) de $95 \%$, com cálculo das razões de chances ajustadas.

Esta pesquisa seguiu os princípios éticos presentes na Declaração de Helsinque e na Resolução n 196/96 do Conselho Nacional de Saúde. Os protocolos de pesquisa foram avaliados e aprovados pelo Comitê de Ética em Pesquisa com Seres Humanos da Universidade Estadual do Sudoeste da Bahia.

\section{Resultados}

A amostra do estudo constituiu-se de $222 \mathrm{mu}$ lheres idosas, com a média de idade de 70 anos $( \pm 7,27)$, com a amplitude de 60 a 92 anos. A Tabela 1 contempla um resumo das características das idosas conforme as variáveis independentes incluídas na investigação.

Embora tenham sido observadas algumas variações nos grupos etários estabelecidos, a população em geral pode ser descrita com predominância de viúvas, o arranjo familiar caracterizado como sendo multigeracional, sendo de baixo nível de escolaridade e com a maioria dos participantes apresentando baixa classificação socioeconômica.

O tabagismo foi referido como um hábito do passado por $37,4 \%(n=83)$, mas no presente apenas $6,3 \%(n=14)$ permanecem com tal comportamento. Já para a prática de atividades físicas foi observado fenômeno inverso, sendo que no passado apenas $18,5 \%(n=41)$ realizavam atividades físicas no momento de lazer, e no presente tal hábito foi reportado por 50,9\% $(n=113)$.

$\mathrm{O}$ estado nutricional verificado pelo IMC demonstrou que $64 \%(n=142)$ estavam classificadas na condição de sobrepeso ou obesidade (IMC superior a $24,9 \mathrm{~kg} / \mathrm{m}^{2}$ ). Os sintomas depressivos foram relatados por $18,9 \%(n=42)$ das participantes, sendo que a alteração do estado cognitivo foi identificada em 75,2\% $(n=167)$. E ainda o uso de medicamentos foi referido por $72,2 \%$ $(n=167)$, sendo que $23,9 \%(n=53)$ fazem uso de três ou mais medicamentos/dia.

Em relação às doenças autorreferidas, as relativas ao aparelho circulatório, sistema osteomuscular, doenças dos olhos, ouvidos e metabólicas foram respectivamente as mais referidas pelas idosas entrevistadas.

Outras condições de saúde foram referidas, a exemplo da ocorrência de quedas nos últimos três meses, reportadas por $21,2 \%(n=47)$, e de internações nos últimos seis meses, $15,8 \%$ $(n=35)$. Há ainda que se ressaltar o fato de $46,8 \%$

Tabela 1. Distribuição de variáveis sociodemográficas, aspectos comportamentais, saúde física e mental de idosas do município de Jequié (BA).

\begin{tabular}{|c|c|c|}
\hline Característica & $\mathbf{n}$ & $\%$ \\
\hline \multicolumn{3}{|l|}{ Idade (anos) } \\
\hline $60-69$ & 115 & 51,8 \\
\hline $70-79$ & 85 & 38,3 \\
\hline 80 ou mais & 22 & 9,9 \\
\hline \multicolumn{3}{|l|}{ Estado civil } \\
\hline Solteira/divorciada & 43 & 19,4 \\
\hline Casada ou vivendo com o parceiro & 73 & 32,9 \\
\hline Viúva & 106 & 47,7 \\
\hline \multicolumn{3}{|l|}{ Arranjo familiar } \\
\hline Multigeracional (residir com filhos e netos) & 100 & 45 \\
\hline \multicolumn{3}{|l|}{ Nível socioeconômico } \\
\hline Classes D e E & 160 & 72,1 \\
\hline \multicolumn{3}{|l|}{ Tempo de escolarização } \\
\hline$<2$ anos & 121 & 54,5 \\
\hline Uso de medicamentos & 167 & 75,2 \\
\hline \multicolumn{3}{|l|}{ Quantidade de medicamentos } \\
\hline Uso de dois ou mais & 110 & 49,6 \\
\hline \multicolumn{3}{|l|}{ Comportamentos } \\
\hline Sobrepeso/obesidade & 142 & 64 \\
\hline Tabagista (passado) & 83 & 37,4 \\
\hline Tabagista (presente) & 14 & 6,3 \\
\hline Atividades físicas (passado) & 41 & 18,5 \\
\hline Atividades físicas (presente) & 113 & 50,9 \\
\hline Sintomas depressivos & 42 & 18,9 \\
\hline Déficit cognitivo & 167 & 75,2 \\
\hline \multicolumn{3}{|l|}{ Doenças autorreferidas } \\
\hline Aparelho circulatório & 167 & 75,2 \\
\hline Sistema osteomuscular & 159 & 71,6 \\
\hline Doenças dos olhos & 119 & 53,6 \\
\hline Doenças do ouvido & 93 & 41,9 \\
\hline Metabólicas & 50 & 22,5 \\
\hline Aparelho geniturinário & 22 & 9,9 \\
\hline Aparelho digestivo & 16 & 7,2 \\
\hline Aparelho respiratório & 15 & 6,8 \\
\hline Sistema nervoso & 10 & 4,5 \\
\hline Neoplasias & 4 & 1,8 \\
\hline Infecciosas e parasitárias & 4 & 1,8 \\
\hline Hematopoéticos & 2 & 0,9 \\
\hline \multicolumn{3}{|l|}{ Outras condições de saúde } \\
\hline Ocorrência de quedas & 47 & 21,2 \\
\hline Internações nos últimos seis meses & 35 & 15,8 \\
\hline \multicolumn{3}{|l|}{ Saúde percebida } \\
\hline Ruim & 41 & 18,5 \\
\hline Regular & 106 & 47,7 \\
\hline Boa ou excelente & 75 & 33,8 \\
\hline \multicolumn{3}{|l|}{ Incapacidade funcional } \\
\hline Dependência moderada/grave nas AIVDs & 104 & 46,8 \\
\hline
\end{tabular}


$(\mathrm{n}=104)$ indicarem algum tipo de dependência do tipo moderada e/ou grave na realização das atividades instrumentais da vida diária (AIVDs). No entanto, a saúde percebida indicou que 33,8\% $(\mathrm{n}=75)$ possuíam uma percepção otimista da condição de saúde e apenas 18,5\% ( $\mathrm{n}=41)$ reportaram uma saúde em condição "ruim".

Na Tabela 2, estão dispostas as variáveis que se mostraram relacionadas com a dependência do tipo moderada ou grave nas AIVDs (incapacidade funcional). $\mathrm{O}$ aumento da idade e a condição de viuvez referida pelas entrevistadas em relação às que referiram serem solteiras ou divorciadas foi um fator de risco para a incapacidade funcional.
Já o tempo de escolarização se mostrou relacionado com a incapacidade funcional de modo inverso, em que quanto maior os anos de estudo da pessoa, menor as chances de o indivíduo apresentar dificuldades na realização das AIVDs, assim como para as classes econômicas. As pessoas de baixa condição econômica possuem mais chances de serem incapazes funcionalmente em relação às idosas mais favorecidas economicamente.

Tanto a inatividade física de lazer no período atual quanto a do passado estão relacionada com a incapacidade funcional; o mesmo ocorre com as alterações cognitivas. $\mathrm{O}$ uso de três ou mais medicamentos contínuos também se mostrou associado às incapacidades na realização das AI-

Tabela 2. Análise bruta para mulheres idosas residentes no município de Jequié (BA) em relação ao desfecho dependência moderada/grave nas AIVDs.

\begin{tabular}{|c|c|c|}
\hline Característica & Razão de chances $[95 \%]$ & $\mathbf{P}$ \\
\hline \multicolumn{3}{|l|}{ Idade (anos) } \\
\hline $60-69$ & 1,00 & \\
\hline $70-79$ & $1,5[0,87-2,7]$ & 0,13 \\
\hline 80 ou mais & $3,2[1,21-8,4]$ & 0,01 \\
\hline \multicolumn{3}{|l|}{ Estado civil } \\
\hline Solteira/divorciada & 1,00 & \\
\hline Casada ou vivendo com o parceiro & $1,6[0,73-3,55]$ & 0,23 \\
\hline Viúva & $2,5[1,19-5,26]$ & 0,01 \\
\hline Tempo de escolarização (anos) & $0,86[0,78-0,95]$ & 0,00 \\
\hline \multicolumn{3}{|c|}{ Nível socioeconômico (Classes econômicas) } \\
\hline Classes $A_{1}, A_{2}, B_{1}, B_{2}$ e $C$ & 1,00 & \\
\hline $\mathrm{D}$ & $1,12[0,61-2,06]$ & 0,70 \\
\hline $\mathrm{E}$ & $3,36[1,21-9,26]$ & 0,01 \\
\hline \multicolumn{3}{|l|}{ Comportamentos } \\
\hline \multicolumn{3}{|l|}{ Atividades físicas (passado) } \\
\hline Sim & 1,00 & \\
\hline Não & $3,92[1,77-8,69]$ & 0,00 \\
\hline \multicolumn{3}{|l|}{ Atividades físicas (presente) } \\
\hline Sim & 1,00 & \\
\hline Não & $1,78[1,04-3,03]$ & 0,03 \\
\hline \multicolumn{3}{|l|}{ Função cognitiva } \\
\hline Sem alteração & 1,00 & \\
\hline Com alteração & $4,39[2,16-8,93]$ & 0,00 \\
\hline \multicolumn{3}{|l|}{ Quantidade de medicamentos } \\
\hline Até um medicamento & 1,00 & \\
\hline Dois medicamentos & $1,47[0,77-2,79]$ & 0,24 \\
\hline Mais de dois medicamentos & $2,32[1,18-4,53]$ & 0,01 \\
\hline \multicolumn{3}{|l|}{ Doença autorreferidas } \\
\hline \multicolumn{3}{|l|}{ Hipertensão arterial } \\
\hline Ausência & 1,00 & \\
\hline Presença & $2,09[1,18-3,71]$ & 0,01 \\
\hline \multicolumn{3}{|l|}{ Outras condições de saúde } \\
\hline \multicolumn{3}{|l|}{ Hospitalização nos últimos seis meses } \\
\hline Ausência & 1,00 & \\
\hline Presença & $2,5[1,17-5,3]$ & 0,01 \\
\hline
\end{tabular}


VDs, do mesmo modo que a presença de hipertensão arterial e a ocorrência de hospitalização.

Os resultados demonstrados na Tabela 2 serviram para determinar a ordenação dos blocos (modelos) e de variáveis a serem inseridas na análise multivariada hierarquizada apresentada na Tabela 3, de modo a explicar melhor as interações entre as características informadas pelas idosas do estudo.

$\mathrm{Na}$ Tabela 3, dentre as características sociodemográficas analisadas de forma interagidas no primeiro bloco, apenas o aumento da idade cronológica e o baixo tempo de escolarização se mantiveram relacionados com o declínio da capacidade funcional. No segundo bloco analisado, referente às características de saúde e controlado para o bloco anterior, a única variável que permaneceu no modelo foi a ocorrência de hospitalização nos últimos seis meses. Já no terceiro bloco, foi identificado que as pessoas que não realizaram práticas de atividades físicas de lazer no passado e apresentavam alterações cognitivas possuíam mais chances para incapacidades na realização das AIVDs, em relação aos que tiveram experiência com práticas de atividades físicas e ausência de déficit cognitivo, respectivamente.

\section{Discussão}

A preservação da capacidade funcional proporciona ao idoso a realização das atividades cotidianas de forma independente e ainda a participação em atividades sociais, que se configura um aspecto preponderante na saúde mental em idades mais avançadas.

Apesar de haver diferenças nos critérios adotados na classificação da incapacidade funcional, levantamentos populacionais realizados tanto em países desenvolvidos ${ }^{13,14}$ como em desenvolvimento $^{15,16}$ indicam índices de incapacidade na realização das AIVDs bem menores do que apresentados na presente investigação. Esse fato reforça a necessidade de se explorarem os fatores associados à condição de incapacidade na realização das atividades do dia a dia em idosos residentes em comunidades de baixa renda no Nordeste brasileiro.

A associação de baixos níveis socioeconômicos com piores condições de saúde tem sido documentada, por meio de estudos populacionais, em diversos grupos de idade e em diferentes áreas do mundo ${ }^{15-17}$. O baixo poder aquisitivo está relacionado a uma série de condições negativas, podendo contribuir para a perda da autonomia
Tabela 3. Análise ajustada para mulheres idosas em relação ao desfecho dependência moderada/grave nas AIVDs, município de Jequié (BA).

\begin{tabular}{lcc}
\hline & \multicolumn{2}{c}{ Modelo 1 } \\
\cline { 2 - 3 } Característica & $\begin{array}{c}\text { Razão de } \\
\text { chances [95\%] }\end{array}$ & p \\
\hline Bloco sociodemográfico & & \\
Idade (anos) & $1,04[1,00-1,09]$ & 0,01 \\
Tempo de escolarização & $0,86[0,78-0,95]$ & 0,00 \\
Bloco saúde & & \\
Hospitalização nos últimos & & \\
seis meses & 1,00 & \\
$\quad$ Ausência & & \\
$\quad$ Ocorrência & $2,49[1,14-5,40]$ & 0,02 \\
Bloco comportamentos & & \\
relacionados à saúde & & \\
Atividades físicas (passado) & 1,00 & \\
Sim & $4,03[1,67-9,73]$ & 0,00 \\
Não & & \\
Função cognitiva & 1,00 & \\
Sem alteração & $4,46[1,94-10,55]$ & 0,00 \\
Com alteração &
\end{tabular}

"Ajustado pelo bloco sociodemográfico; ${ }^{* *}$ Ajustado pelos blocos sociodemográficos e de saúde.

funcional, a exemplo da baixa escolaridade, escassez de emprego, condições precárias de saúde, dentre outros fatores. As pessoas de condições menos favorecidas economicamente possuem 3,3 vezes mais chances de apresentar alguma dependência do tipo moderada a grave na realização das AIVDs em relação às de classes economicamente mais favorecidas.

Em estudos epidemiológicos direcionados à identificação de fatores de risco individual, a magnitude do impacto dos fatores socioeconômicos sobre a saúde tem sido menos enfatiza$\mathrm{da}^{18}$. O baixo nível socioeconômico não é considerado entre os fatores modificáveis, sendo frequentemente omitido nos planejamentos estratégicos de prevenção ${ }^{19}$.

O tempo de escolaridade foi associado à capacidade funcional, evidenciando que quanto mais elevado o tempo de escolaridade, maiores são as chances de a pessoa se manter autônoma. Esses resultados são reforçados por resultados de outros levantamentos populacionais ${ }^{15,20}$, que encontraram maiores riscos para o declínio da capacidade funcional, em categorias de escolarização mais baixa. O nível de escolaridade atingido pelo idoso parece ser determinante do tipo de ocupação e do nível de renda pelos quais passou 
no decorrer do tempo ${ }^{15}$. Talvez esse aspecto explique o fato de a inserção da variável "tempo de escolaridade" no primeiro modelo da análise hierarquizada ter sido determinante para que o fator "classificação econômica" não se mantivesse como aspecto relacionado à incapacidade funcional das mulheres idosas. Do mesmo modo, parece ser mais provável que uma pessoa com níveis de escolaridade mais baixa esteja exposta a piores condições de trabalho, com salários menores, do que uma pessoa com um nível mais alto de escolaridade, apesar de não ter sido encontrada associação significativa que demonstrasse qualquer tipo de relação entre situação ocupacional e dependência funcional.

A variável "faixa etária" constituiu-se em determinante da capacidade funcional, confirmando outros estudos ${ }^{14,15,21}$. Há uma associação da faixa etária com a dependência funcional, sendo que à medida que aumenta a idade cronológica também ocorre um aumento na chances de se apresentar alguma dependência funcional. Porém, essa associação foi discreta, condição justificada pelo aspecto de que a idade avançada não explica, por si só, a incapacidade funcional, necessitando-se do conhecimento sobre a mediação de fatores ambientais e de características correlacionadas com a idade avançada ${ }^{15}$. Talvez por esse motivo a idade tenha permanecido no modelo explicativo final deste estudo com um fator de explicação baixa para a determinação da capacidade funcional.

Quando analisado o estado civil atual entre as idosas, esta variável apresentou-se associada à capacidade funcional, sendo que o estado de viuvez entre as idosas representa um risco de 2,5 vezes mais chances de apresentar algum tipo de dependência em relação às que são solteiras. Mas tal relação não se manteve na análise multivaria$\mathrm{da}$, em que os fatores aumento de idade e baixo nível econômico sobrepuseram-se em relação às demais variáveis estudadas neste estudo como determinantes sociodemográficas da incapacidade funcional.

Dentre os problemas de saúde reportados, os relacionados com o aparelho circulatório foram os mais prevalentes, sendo que dentre tais problemas a hipertensão arterial se mostrou com uma razão de chances de 2,0 vezes mais para se ter dependência nas AIVDs em comparação com as pessoas que não referiram possuir hipertensão arterial, apesar de os problemas de saúde reportados não permanecerem no modelo final explicativo da incapacidade funcional. As doenças, em geral, agem como um fator de risco para a dependência funcional, o que é reforçado por outros estudos ${ }^{21,22}$.

O fator referente à hospitalização nos últimos seis meses apresentou-se como um risco de 2,7 vezes mais chances de algum tipo de dependência, em relação aos que não foram hospitalizados. Porém, a hospitalização na realidade não é, em si, risco para o declínio da capacidade funcional, mas um indicador da ocorrência de tipos graves de doenças que demandam cuidados hospitalares ${ }^{15}$.

Os dados descritivos indicam ainda a elevada prevalência de pessoas com sobrepeso ou obesidade, que estavam classificadas com o IMC superior a $24,9 \mathrm{~kg} / \mathrm{m}^{2}$. Tal característica da população posiciona os sujeitos do estudo em uma condição de risco, tendo em vista os agravos ocasionados pelo excesso de peso á saúde das pessoas ${ }^{23,24}$. Há a necessidade de intervenções em comunidades com baixo poder aquisitivo, pois o Nordeste, assim como outras regiões pobres do país, vem passando por um fenômeno denominado de transição nutricional, em que o problema de escassez alimentar tem sido substituído pelo excesso de alimentos ricos em gordura, o que gera um aumento de sobrepeso e obesidade ${ }^{25}$.

A atividade física praticada nos momentos de lazer ao longo da vida e as alterações cognitivas parecem ser determinantes na classificação do indivíduo em capaz ou incapaz para a realização das AIVDs. As idosas que mostraram alguma alteração cognitiva possuíam 4,4 vezes mais chances de apresentarem alguma dependência funcional, mesmo quando controladas para a realização de atividades físicas de lazer no passado e as variáveis de saúde e sociodemográficas.

Há que se considerar a interação já bem documentada na literatura quanto aos benefícios da prática de atividades físicas, que se mantém relacionada com a condição cognitiva ${ }^{25,26}$. Tal relação necessita ainda de que os mecanismos biológicos que estão envolvidos na interação desses componentes sejam mais bem caracterizados. Por um lado, é de entendimento geral que a conservação de parâmetros funcionais melhore a vascularização, a utilização de unidades neuromotoras e o desenvolvimento da aptidão funcional ${ }^{27}$; por outro lado, o domínio social é estimulado com a preservação da comunicação entre as pessoas ${ }^{28}$, sendo um elo entre o domicílio e a comunidade. Os aspectos destacados podem ser considerados determinantes na preservação da condição de realizar as tarefas instrumentais da vida cotidiana.

A manutenção da função cognitiva congrega uma série de fatores que pode ter sobreposto o 
efeito "de associação" de outras características estudadas; em decorrência, as características idade, tempo de escolaridade e até mesmo algumas doenças estão estreitamente vinculadas com a função cognitiva do indivíduo ${ }^{25,26}$.

O delineamento transversal, apesar da conveniência quanto à redução do tempo e do custo na coleta de informações, apresenta limitações em relação às inferências causais, pois as exposições e o desfecho são coletados em um mesmo momento. No caso específico do presente estudo, as relações entre a incapacidade funcional e os fatores sociodemográficos, saúde física e mental podem ter sido afetadas por esse viés. Porém, mesmo com as potenciais limitações do delineamento transversal, os dados analisados parecem suportar as evidências de modelos explicativos de geração das incapacidades funcionais na população idosa.

Em razão de as características descritivas apresentadas serem similares aos indicadores sociais do Nordeste brasileiro, que indicam baixos estratos, de classe economicamente desprovida, é permitido sugerir a generalização dos resultados encontrados neste estudo.

Por fim, os dados encontrados neste estudo sugerem que a população de mulheres idosas residentes em Jequié, um município de pequeno porte no Nordeste do Brasil, possuem elevada prevalência de limitações na realização das atividades instrumentais da vida diária. Assim, os resultados apresentados demonstram ser necessários à implementação de ações públicas que possam favorecer a redução e/ou retardamento das incapacidades. Essas intervenções devem ser específicas a determinadas faixas etárias, ser direcionadas ao aumento da escolarização e melhoria da condição econômica e na assistência aos idosos hospitalizados, além do incentivo ao aumento da participação em práticas de atividades físicas e à preservação da saúde mental.

\section{Colaboradores}

JS Virtuoso Júnior participou da elaboração do artigo nas fases de concepção, delineamento, análise/interpretação dos dados e redação do artigo; RO Guerra participou das fases de análise/interpretação dos dados e revisão crítica da redação final.

\section{Agradecimentos}

À Fundação de Amparo à Pesquisa do Estado da Bahia (Fapesb), pelo apoio concedido na realização deste trabalho. 


\section{Referências}

1. Lunney JR, Lynn J, Foley DJ, Lipson S, Guralnik JM. Patterns of functional decline at the end of life. JAMA 2003; 289:2387-2392.

2. Arthanat S, Nochajski SM, Stone J. The international classification of functioning, disability and health and its application to cognitive disorders. Disabil Rehabil 2004; 26(4):235-245.

3. Béland F, Zunzunegui MV. La salud y las incapacidades funcionales: elaboración de um modelo causal. Rev. Gerontol 1995; 5:259-273.

4. Associação Nacional de Empresas de Pesquisa. Critério de Classificação Econômica Brasil [site na Internet]. [acessado 2003 fev]. Disponível em: http:// www.abep.org/codigosguias/ABEP_CCEB.pdf

5. Organização Mundial da Saúde (OMS). Classificação estatística internacional de doenças e problemas relacionados à saúde: CID-10. São Paulo: EDUSP; 1994.

6. Lawton MP, Brody EM. Assessment of older people: self-maintaining and instrumental activities of daily living. Gerontologist 1969; 9:179-186.

7. Pfeiffer E. A short portable mental status questionnaire for the assessment of organic brain deficit in elderly patients. J Am Geriatr Soc 1975; 23:433-441.

8. Shah A, Herber TR, Lewis S, Mahendran R, Platt J Bhattacharyya B. Screening for depression among acutely ill geriatric inpatients with a short geriatric depression scale. Age Ageing 1997; 26:217-221.

9. Blay SL, Ramos LR, Mari JJ. Validity of a Brazilian version of the Older Americans Resources and Services (OARS) Mental Health Screening Questionnaire. JAGS 1988; 36:687-692.

10. Almeida OP, Almeida SA. Confiabilidade da versão brasileira da escala de depressão em geriatria (GDS) versão reduzida. Arq Neuropsiquiatr 1999; 57:421426.

11. Hosmer DM, Lemeshow S. Applied logistic regression. New York: John Wiley \& Sons; 1989.

12. Victora CG, Huttly SR, Fuchs SC, Olinto MTA. The role of conceptual frameworks in epidemiological analysis: a hierarchical approach. Int J Epidemiol 1997; 26:224-227.

13. Boyle PA, Buchman AS, Wilson RS, Bienias JL, Bennett DA. Physical activity is associated with incident disability in community-based older persons. J Am Geriatr Soc 2007; 55(2):195-201.

14. Alquézar AL, Aranda ER, Sánchez AS, Herrero JCG. Capacidad funcional para las actividades de la vida diária em las personas mayores que acudieron a centros de convivência em Zaragoza capital em 2005. Rev Esp Salud Publica 2007; 81: 625-636.

15. Rosa TEC, Benicio MHD, Latorre MRDO, Ramos LR. Fatores determinantes da capacidade funcional entre idosos. Rev Saude Publica 2003; 37(1):40-48.

16. Monge MB, Mayo EJG, Robledo LMG, Jaimes AM. Dependencia functional y enfermedades crónicas en ancianos mexicanos. Salud Pública Méx 2007; 49(Supl.4):459-466.
17. Lima Costa MF, Barreto S, Giatti L, Uchoa E. Desigualdade social e saúde entre idosos brasileiros: um estudo baseado na Pesquisa Nacional por Amostra de Domicílios. Cad Saude Publica 2003; 19(3):745757.

18. Drachler ML, Côrtes SMV, Castro JDC, Leite JCC. Proposta de metodologia para selecionar indicadores de desigualdade em saúde visando definir prioridades de políticas públicas no Brasil. Cien Saude Colet 2003; 8(2):461-470.

19. Guralnik JM, Leveille SG. Race, ethnicity, and health outcomes unraveling the mediating role of socioeconomic status. Am J Epidemiol 1997; 137:845-857.

20. Sulander T, Martelin T, Sainio P, Rahkonen O, Nissinen A, Uutela A. Trends and educacional disparities in functional capacity among people aged 65-84 years. Int J Epidemiol 2006; 35(5):1261-1263.

21. Rejeski WJ, Focht BC. Aging and physical disability: on integrating group and individual counseling with the promotion of physical activity. Exercise and Sport Sciences Reviews 2002; 30(4):166-170.

22. Verbrugge LM, Jette A. The disablement process. Social Science in Medicine 1994; 38:1-14.

23. Intorre F, Maiani G, Cuzzolaro M, Simpson EE, Catasta G, Ciarapica D, Mauro B, Toti E, Zaccaria M, Coudray C, Corelli S, Palomba L, Polito A. Descriptive data on lifestyle, anthropometric status and mental health in Italian elderly people. J Nutr Health Aging 2007; 11(2):165-174.

24. Acuña K, Cruz T. Avaliação do estado nutricional de adultos e idosos e situação nutricional da população brasileira. Arq Bras Endocrinol Metab 2004; 48(3):345-361.

25. Yaffe K, Barnes D, Nevitt M, Lui L-Y, Covinsky K. A prospective study of physical activity and cognitive decline in elderly women: women who walk. Arch Intern Med 2001; 161:1703-1708.

26. Atkinson HH, Cesari M, Kritchevsky SB, Penninx BW, Fried LP, Guralnik JM, Williamson JD. Predictors of combined cognitive and physical decline. $J$ Am Geriatr Soc 2005; 53(7):1197-1202.

27. Collins K, Brenda L, Rooney BL, Smalley KJ, Havens $S$. Functional fitness, disease and independence in community-dwelling older adults in Western Wisconsin. Wisconsin Medical Journal 2004; 103(1):42-48.

28. Ramos MP. Apoio social e saúde entre idosos. Sociologias 2002; 7:156-175.

Artigo apresentado em 10/05/2008

Aprovado em 15/01/2009

Versão final apresentada em 15/02/2009 